Review

Demand Side Management on the Chile Industry: Learning From the German Case

\title{
Gestión de la Demanda en la Industria de Chile: Aprendiendo del
}

\section{Caso Alemán}

\section{Y. Masip ${ }^{* 1}$, A. Poque ${ }^{1}$, M. Valín ${ }^{1}$, L. Ramírez ${ }^{2}$, J. Valdés ${ }^{1}$}

${ }^{1}$ Pontificia Universidad Católica de Valparaíso (Valparaíso-Chile), ${ }^{2}$ Technische Hochschule Deggendorf (Freyung-Alemania).

${ }^{*}$ Correspondence e-mail:yunesky.masip@pucv.cl

Received: 29/06/2019. Modified: 18/09/2019. Accepted: 09/10/2019

\begin{abstract}
Context: Both pioneer countries and those that have recently begun an energy transition towards renewable energy require high levels of flexibility in their electrical systems. This article reviews the Demand Side Management (DSM) application as a tool to provide flexibility, and then, to achieve renewable energy penetration objectives.
\end{abstract}

Method: A systematic mini-review of the literature has been done to review the origin and evolution of the concept of DSM in German and Chilean industry, at the same time, to make a parallel and comparison between them.

Results: Both countries have difficulties in taking advantage of the demand management potential in the industry, the lack of promoting policies and the lack of specific regulatory frameworks are some of the causes.

Conclusions: It is necessary to speed up attention to the management of demand, it is important that the academy contributes with estimates of potential, it also needs public policies that encourage the use of this tool.

Keywords: Demand side management, renewable sources of energy, flexibility of the electrical system, international cooperation, energy policy.

Acknowledgments: The authors wish to thank the National Commission for Scientific and Technological Research of Chile (CONICYT) and the German Ministry of Education and Research (BMBF) for their support, which, in the framework of project BMBF150075: "Increasing renewable energy penetration in industrial production and grid integration through optimized CHP energy dispatch scheduling and demand side management", have made possible the development of this article. In addition, to the Deutsche Gesellschaft für Internationale Zusammenarbeit (GIZ) $\mathrm{GmbH}$, for all the support provided through the Energy Program in Chile.

Language: Spanish.

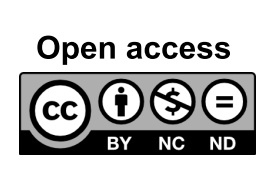

Cite this work as: Y Masip, A. Poque, M. Valín, L. Ramírez and J. Valdés., "Demand Side Management on the

Chile Industry: learning from the German case", Ingeniería, vol. 24, no.3, pp. 235-251, Sep-Dec. 2019.

(C) The authors; reproduction right holder Universidad Distrital Francisco José de Caldas.

DOI:https://doi.org/10.14483/23448393.14709 


\section{Resumen}

Contexto: Tanto países pioneros como aquellos que recientemente han comenzado una transición energética hacia las energías renovables requieren altos niveles de flexibilidad en sus sistemas eléctricos. Este artículo revisa la aplicación de la gestión de la demanda (DSM, por sus siglas en inglés) como herramienta para proveer flexibilidad y lograr objetivos de penetración de fuentes renovables de energía.

Método: Se ha hecho una revisión sistemática de la literatura para revisar el origen y evolución del concepto de DSM en la industria alemana y chilena, realizando al mismo tiempo un paralelo y comparación entre ambas experiencias.

Resultados: Ambos países registran dificultades a la hora de aprovechar el potencial de gestión de la demanda en la industria, la falta de políticas promotoras y la carencia de marcos regulatorios específicos son algunas de las causas.

Conclusiones: Se requiere agilizar la atención a la gestión de la demanda; además, es importante que la academia contribuya con estimaciones de potencial en tanto se precisan políticas públicas que fomenten el aprovechamiento de esta herramienta.

Palabras clave: Cooperación internacional, flexibilidad del sistema eléctrico, fuentes renovables de energía, gestión de la demanda, política energética.

Agradecimientos: Los autores desean agradecer el apoyo de la Comisión Nacional de Investigación Científica y Tecnológica de Chile (CONICYT) y al Ministerio de Educación e Investigación de Alemania (BMBF), los cuales, en el marco del proyecto BMBF150075: "Increasing renewable energy penetration in industrial production and grid integration through optimized CHP energy dispatch scheduling and demand side management", han hecho posible el desarrollo de este artículo. Asimismo, a Deutsche Gesellschaft für Internationale Zusammenarbeit (GIZ) $\mathrm{GmbH}$, por todo el apoyo brindado a través del Programa de Energía en Chile.

Idioma: Español

\section{Introducción}

En los últimos años, Chile ha experimentado una notable transformación en su matriz energética dada la potente incorporación de fuentes renovables de energía. De acuerdo con el informe "Tendencias globales en la inversión en energías renovables 2019”, publicado antes de la Cumbre sobre la Acción Climática de la ONU, Chile se coloca en el puesto diecinueve en inversiones en capacidad de energías renovables durante esta década, ello con un balance de US 14000 millones entre 2010 y la primera mitad de 2019, lo cual lo deja, además, en el tercer lugar a nivel de Sudamérica en este sentido. Asimismo, la intermitencia de la energía solar y eólica plantea nuevos retos relacionados con la estabilidad y la confiabilidad del sistema [1], ya que la estocasticidad del recurso viento y sol dificultan a los operadores del sistema la tarea de mantener el equilibrio entre oferta y demanda de energía [2].

Una forma de proveer la estabilidad y flexibilidad es a través de gestión de la demanda (DSM, por sus siglas en inglés) [3]. DSM se refiere en general a tecnologías, acciones y programas que buscan administrar o disminuir el consumo de energía desde el lado de la demanda a fin de reducir los desembolsos totales del sistema o contribuir al logro de objetivos de política tales como la reducción de emisiones o equilibrar la oferta y demanda [4]. El concepto de DSM se basa en un nuevo 
modo de realizar la gestión de la demanda, permitiendo al cliente ser responsable de esta. La idea de utilizar DSM en la industria para ofrecer flexibilidad a sistemas eléctricos con alta penetración de energías renovables no convencionales (ERNC) no es nueva, incluso Alemania, país pionero de la transición energética, tiene trabajo por hacer para aprovechar sus potenciales de DSM [5].

Este artículo muestra los principales avances relativos a DSM en materia de política energética para el caso chileno, al tiempo que desarrolla un análisis comparativo con la experiencia alemana. El objetivo es proponer recomendaciones para enfrentar los próximos desafíos del sector, específicamente aquellos relacionados con DSM en la industria, teniendo en cuenta los aciertos y desaciertos del caso alemán. El resto del artículo está estructurado de la siguiente manera: en sección 2 se describe la metodología empleada para el desarrollo de este trabajo, en 3.1 se hace una revisión del desarrollo histórico y conceptos básicos de DSM, mientras que en las secciones 3.2 y 3.3 se presentan los avances respecto de DSM en Alemania y Chile. En la sección 4 se discuten los retos para la integración de DSM en la industria y en sección 5 se aúnan conclusiones.

\section{Metodología}

Con el objeto de revisar el origen y evolución del concepto de DSM en la industria alemana y chilena, realizando al mismo tiempo un paralelo entre ellas, se ha hecho una revisión sistemática de la literatura para desarrollar discusión al respecto. El desarrollo metodológico consiste en una búsqueda de documentos relativos a DSM en Alemania y Chile, seleccionando aquellos que refieren a la aplicación industrial, y luego, otorgando mayor valoración a los artículos y literatura de política energética. Posteriormente, la información extraída para la elaboración de esta revisión se usará en procura de capturar los principales hitos que han permitido el avance de DSM en ambos países. Finalmente, se desarrolla una breve discusión en torno a los retos para la explotación del potencial de DSM y, asimismo, se propone recomendaciones para el caso chileno, teniendo en cuenta el avance de Alemania, éxitos y desaciertos de su experiencia. En la Figura 1, se muestra una categorización de las temáticas abordadas en la revisión bibliográfica.

\section{Desarrollo}

\subsection{Desarrollo histórico y conceptos básicos acerca de gestión de la deman- da}

Las primeras nociones de DSM datan de 1960 en Nueva Zelanda y Europa, donde se gestionaba la carga en empresas; sin embargo, el término DSM fue acuñado por primera vez por Clark Gellings [6] e introducido formalmente en política energética en 1978 por la National Energy Conservation Policy Act y la Public Utility Regulatory Policy Act de los Estados Unidos. Luego, durante la década de 1980 y 1990 los programas de DSM crecieron, llegando a ahorrar $260000 \mathrm{GWh}$ entre 1989 y 1995 en Estados Unidos; no obstante, desde 1995 declinaron y solo volvieron a reactivarse entre 2000 y 2010 a partir del interés por enfrentar el cambio climático y garantizar la seguridad energética [7]. En Europa, los programas de DSM recién comenzaron a cobrar interés en 1990, principalmente motivados por el cambio climático [7]; en China, el concepto de DSM ya se discute también desde esa década [8]. 


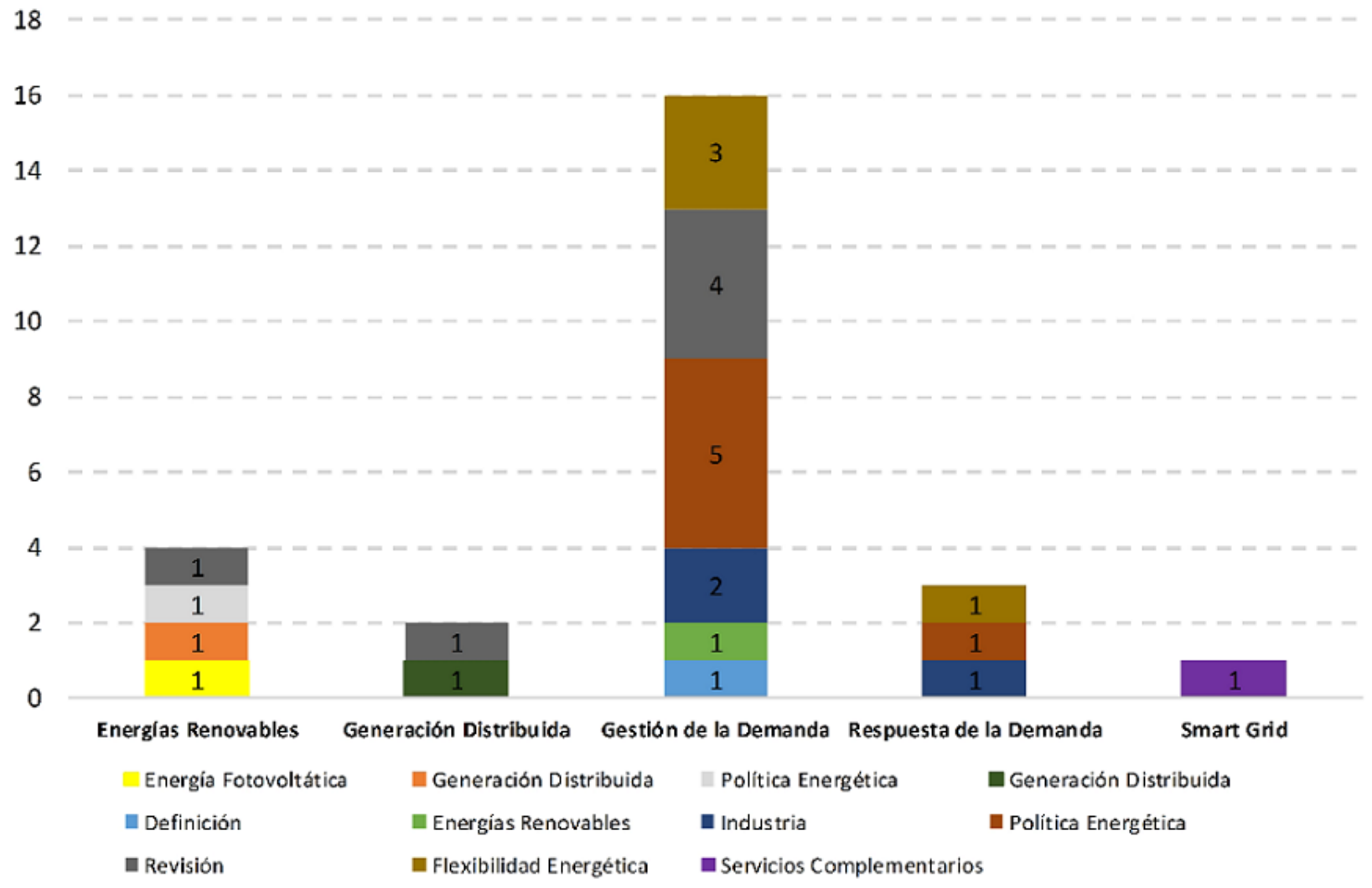

Figura 1. Revisión de la literatura.

DSM es un concepto amplio estudiado por múltiples autores [7], [9]-[13]. En la Figura 2, se puede observar un esquema de dicha complejidad en su concepto fundamental y más amplio, tal como lo propone [7]. La complejidad inicia con la categorización de las diferentes opciones de DSM que, por lo menos, incluye la eficiencia energética, el respaldo de generación in situ y la respuesta de la demanda; estas categorías se subdividen nuevamente en subcategorías que abarcan un amplio campo de acción, el cual no puede ser afrontado de manera unidireccional, sino que depende de múltiples actores. El desarrollo histórico ha mostrado que son variadas las políticas que permiten aplicar las distintas categorías de DSM, estas incluyen desde medidas regulatorias que obligan a las empresas distribuidoras a cumplir con ciertos niveles de conservación energética, hasta campañas informativas para usuarios acerca de generación en sitio y eficiencia energética.

Las medidas que pueden contribuir a que la industria productiva haga DSM se encuentran distribuidas en cuatro tipos de políticas, estas incluyen: la implementación de estándares de manejo de energía (como ISO 50001), la difusión de buenas prácticas, el financiamiento directo para programas en investigación y desarrollo, la simplificación de las condiciones para la participación de las industrias en los mercados energéticos y la implementación de esquemas tarifarios como la definición de precios de la electricidad en tiempo real [14]. 


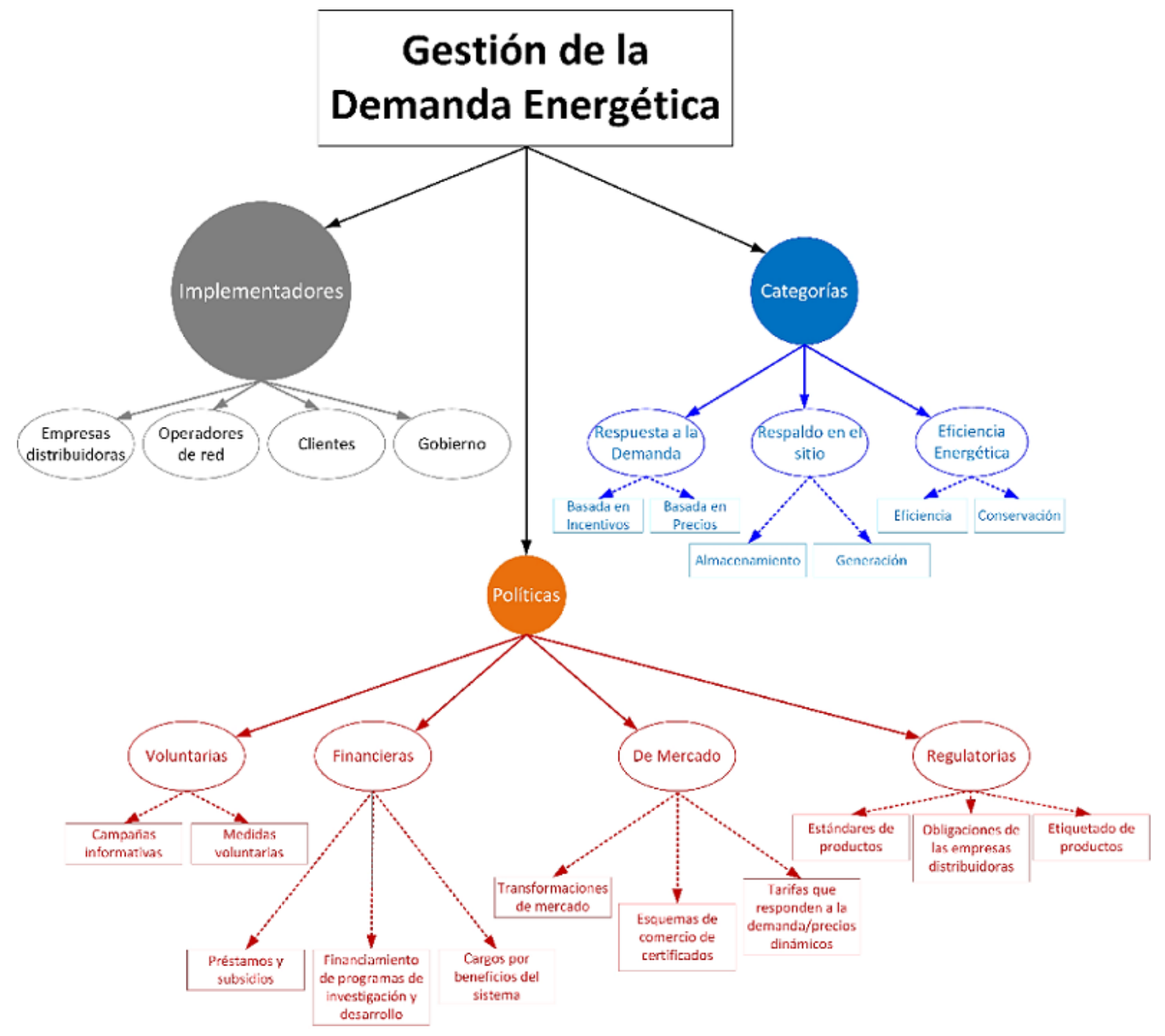

Figura 2. Gestión de la demanda energética y sus componentes bajo los principios de la gestión desde el lado de la demanda.

Fuente: elaboración propia basada en [9].

\subsection{Gestión de la demanda en Alemania}

Alemania es reconocida por ser una de las primeras naciones que adoptó una política de energía limpia con alto porcentaje de ERNC en la matriz energética [15]. Este país ha seguido un largo camino donde las tecnologías, las regulaciones y las prioridades han cambiado considerablemente desde 1991, cuando apareció la primera ley que permitía el suministro descentralizado de ERNC, hasta hoy, donde más del $30 \%$ de la demanda de electricidad es suministrada por fuentes renovables [16]. El principal instrumento para promover la adopción de ERNC, la ley de fuentes renovables de energía (Erneuerbaren Energien Gesetz o EEG, por sus siglas en alemán), apareció en 2000 y hasta ahora ha recibido cuatro enmiendas [17]. EEG comenzó siendo altamente subsidiaria y protectora para los adoptantes de las ERNC, mientras que en su última versión los generadores de ERNC se han dejado libres para encontrar su propio lugar en el mercado. Al principio, era 
importante brindar garantías para la adopción de nuevas tecnologías no convencionales; actualmente, los esfuerzos se concentran en hacer que el mercado se ajuste a las altas penetraciones de ERNC.

A medida que aumenta la proporción de fuentes de energía renovables intermitentes será necesario aumentar el volumen de las reservas de regulación de frecuencia. Se pueden encontrar nuevas fuentes de reservas en los recursos energéticos distribuidos: cargas controlables, vehículos eléctricos (EV), unidades de generación distribuida, etc. Sin embargo, es necesario adaptar el diseño del mercado de reserva de regulación de frecuencia para permitir que estos recursos participen a través de nuevos actores del mercado conocidos como "agregadores". De esta manera, se deben analizar los mercados o mecanismos de regulación de frecuencia para: identificar barreras de entrada para los agregadores e identificar algunas opciones con el fin superar estas barreras. En este sentido, Borne realiza un estudio de caso de la situación de los cuatro principales mercados europeos de regulación de frecuencia para mostrar cómo este marco podría ser utilizado por los agregadores o los responsables políticos [18].

Actualmente, según ENSO-E (Red Europea de Operadores de Sistemas de Transmisión), la potencia renovable ya supone más de la mitad del parque productor alemán con el $56 \%$ de la potencia total instalada. La tecnología más extendida es la eólica con $59 \mathrm{GW}$ que representa el $27 \%$ de la potencia total del país; también es la tecnología con mayor crecimiento de los últimos años pasando del $20 \%$ en 2015 a un $27 \%$ en la actualidad. La potencia solar fotovoltaica instalada actualmente asciende a $43 \mathrm{GW}$, el $20 \%$ de la potencia nacional. Si bien la potencia fotovoltaica ha aumentado un $16 \%$ en los últimos cinco años, su cuota en el parque de producción global se ha quedado estancada justo por debajo del $20 \%$ [19].

Lund y Müller diferencian entre cuatro tipos de flexibilidad en el sistema eléctrico que garantizan la seguridad del suministro: expansión de la red, almacenamiento de energía, flexibilidad en el lado de la oferta y flexibilidad en el lado de la demanda. La flexibilidad en el lado de la demanda se refiere a la posibilidad de desviarse del perfil de carga planificado. Tales desviaciones están económicamente motivadas por las señales de precios, si un tercero envía una señal a un consumidor flexible, esto se llama respuesta a la demanda (DR). Debido a los altos costos para los sistemas de almacenamiento y la expansión de la red, la flexibilidad en el lado de la demanda jugará un papel importante, ya que ofrece flexibilidad a costos marginales relativamente bajos [19], [20].

Alemania ha avanzado bastante en la implantación de medidas para incrementar la eficiencia energética, sobre todo en lo relativo al respaldo de generación en el sitio. Los resultados se visualizan en el auge de las ERNC y el próspero desarrollo en la instalación de sistemas de almacenamiento que no solo se ve fundamentado en las nuevas reglas de juego para generadores distribuidos, sino a incentivos financieros directos para promocionar el autoconsumo que están en vigencia desde 2013 (“KfW programm 275”, banco alemán gubernamental de desarrollo) [21], [22].

La categoría de DSM "respuesta de la demanda" es en la que menos se ha progresado en Alemania [5]. Se señala que hacen falta acciones del Gobierno y que hay una desconexión con la estrategia nacional de adopción de ERNC. Sin embargo, los avances de la Agencia Nacional de Energía Alemana (DENA, por sus siglas en alemán) muestra que se ha trabajado para que DSM y ERNC puedan competir con los suministradores tradicionales de servicios complementarios (SSCC) o $A n$ - 
cillary Services (AS), mas no con los resultados esperados. Alemania es el único país europeo que ha abierto todos los mercados a DSM, pero la normativa dificulta la participación incluso para las industrias intensivas en energía [23].

El sector de servicios es uno de los grupos objetivo para utilizar la demanda flexible, el cual puede tener un alto impacto en tanto se pueden encontrar supermercados, hoteles y edificios de oficinas en regiones de toda Alemania. Lo anterior podría ser una ventaja con respecto a la compensación de la inestabilidad de la red en cualquier región en comparación con las empresas industriales más enfocadas localmente. Las primeras estimaciones señalan potenciales considerables de DR no utilizados para el sector de servicios; sin embargo, actualmente, hay menos conocimiento sobre las opciones de flexibilidad en el sector de servicios que en la industria, además, las barreras del mercado como la falta de un marco regulatorio adecuado que ofrezca incentivos atractivos evitan que se aprovechen los potenciales [24]. Un estudio realizado en 1000 empresas del sector de servicios en Alemania refleja condiciones marco normativas aún no aptas o faltantes, la baja rentabilidad y la falta de conocimiento sobre los potenciales de flexibilidad y los mecanismos de comercialización parecen ser las principales barreras; no obstante, las condiciones marco que promueven las medidas de respuesta a la demanda están mejorando, por ejemplo, las normas técnicas y las últimas revisiones regulatorias. Lo anterior indica que para aprovechar los potenciales de respuesta a la demanda existentes en el sector de servicios se debe apreciar el valor de la flexibilidad en la demanda, por ejemplo, facilitando la participación en el mercado o creando ofertas resp premiando la flexibilidad de demanda de las empresas [24].

Los sistemas de energía distribuida (DES) a escala local constituyen un nicho prometedor para aprovechar la provisión de energía renovable. Estos DES (microcogeneración, centros multienergéticos) integran fuentes renovables, producción combinada de calor y energía, varios métodos de conversión y almacenamiento de energía y gestión activa del lado de la demanda. Dado su potencial para contribuir a las transiciones de energía, es relevante obtener una mejor comprensión de las condiciones bajo las cuales estos sistemas se difunden. Un estudio realizado sobre las responsabilidades percibidas y la absorción tecnológica prevista de los DES entre diferentes grupos sociales en Suiza, Alemania y Austria, atribuye la responsabilidad de las transiciones del sistema energético al Gobierno nacional y a las grandes empresas de suministro de energía. Los municipios y hogares aún no se consideran relevantes; además, se percibe que el apoyo de los DES es independiente de enmarcar su implementación en relación con diferentes escalas de justificación (cambio climático global, independencia energética nacional o autarquía local) [25].

En el caso del sector residencial, se estima que la utilización de respuesta de la demanda por parte de usuarios residenciales tiene potencial, costos relativamente altos, requiere medidores inteligentes y cambios culturales. La respuesta de la demanda por parte de consumidores residenciales debería tener un rol subordinado con respecto a la respuesta de demanda en la industria. Para esta última, se asume que la adaptación de la infraestructura requerida es más barata en comparación con los volúmenes de energía que se manejan; además, las modificaciones en los procesos productivos se pueden lograr si existen incentivos económicos [26]. De hecho, los requisitos básicos para hacer uso de la respuesta de la demanda que incluyen la instalación de medidores y el levantamiento de datos con respecto a consumos energéticos de subprocesos se llevan ya a cabo mediante certificaciones ISO 50001 y EMAS (Reglamento Comunitario de Ecogestión y Ecoauditoría). En el caso 
de ISO 50001, Alemania contaba en 2016 con más de 90000 empresas certificadas [27]; adicionalmente, empresas con altos consumos energéticos usualmente cuentan con unidades controlables de generación de energía (como plantas de generación combinada de calor y electricidad) que en caso de estar conectadas a la red otorgarían más flexibilidad.

Un trabajo realizado con 185 pilotos para el análisis de capacidades técnicas presentó un sistema de gestión de flexibilidad diseñado con el fin de proporcionar una solución de bajo costo para consumidores residenciales, deseando participar en el equilibrio de la red eléctrica. El sistema pronosticaba continuamente la necesidad de flexibilidad en una red eléctrica e informaba a los consumidores sobre los periodos de gestión de la flexibilidad. Los consumidores podían proporcionar su flexibilidad a un agregador a cambio de una recompensa que dependía del esquema de incentivos seleccionado. La automatización de los eventos de gestión de flexibilidad se proporcionaba mediante la interfaz con los dispositivos y el sistema, ello a través de las tecnologías utilizadas y la arquitectura unificada de comunicación de plataforma abierta. Los resultados indicaron que el enfoque propuesto era apropiado para involucrar a los consumidores en eventos de gestión de la flexibilidad, ya que en promedio los participantes de los pilotos redujeron su carga en un $10 \%$ durante un evento pico [28].

En otro estudio, se determinó que los cinco tipos de procesos industriales con mayor potencial de utilización de respuesta de la demanda (cementos, químicos, aluminio, papeles y metalúrgica) podrían ofrecer hasta $2660 \mathrm{MW}$ de potencia de balance al sistema [3]. Para este potencial, existen tres mercados que permiten su utilización en la red eléctrica alemana [29]: (a) el spot-market (transado en bloques de quince minutos) en las modalidades de un día adelante y para transacciones durante el mismo día; (b) el mercado de reservas primario (con tiempos de respuesta menores a 30 segundos), el secundario (con tiempos de respuesta menores a cinco minutos) y el terciario (con tiempos de respuesta menores a quince minutos); (c) el mercado creado por la ordenanza de cargas interrumpibles (AbLav, por sus siglas en Alemán) con múltiples opciones de participación [30].

El spot-market, en sus dos modalidades, fue pensado en tiempos de generación centralizada y convencional, por lo que las opciones reales de participación de la respuesta de demanda es poca y la participación de la industria se reduce a los casos en los que estas tienen generación propia, controlable y eficiente en función de los costos. Los mercados primario y secundario de reservas requieren tiempos de reacción difíciles de alcanzar en procesos productivos, y aunque hay empresas que participan en estos, la mayor parte de la participación se ha concentrado históricamente en el mercado de reservas terciario [31], [32]. Este último cuenta con una licitación por separado de las potencias negativas y positivas, donde el envío debe corresponder entre el $100 \%$ y $120 \%$ de la potencia solicitada, además de que la disponibilidad no debe ser inferior al $100 \%$ dentro del periodo contractual. Estas reglas se aplican a los recursos de generación y carga sin excepción. La industria química, desde 2009, proveía potencial de balance en el mercado terciario de reservas por un total de $660 \mathrm{MW}$; con el objetivo de aumentar la participación y el perfil de los participantes, aquellos recursos con disponibilidad limitada pueden participar a través de un agregador, pero cada carga debe ser precalificada de manera independiente [31]. De esta manera, el potencial de DSM no solo es promovido por parte de la DENA, sino que los agregadores fomentan la participación entre aquellas industrias que cumplen los requisitos. 
El concepto de agregador hace referencia a un modelo de negocio en el cual un operador independiente ofrece un servicio de gestión de la demanda a diferentes clientes, reuniendo el potencial de flexibilidad de cada uno de ellos para participar en el mercado. Esta externalización permite aprovechar economías de escala, reducir costes e incrementa el potencial de los clientes agregados, explotando complementariedades entre las diferentes instalaciones. La actividad de agregación se basa en la instalación de equipos de acceso remoto que conectan la instalación eléctrica con el agregador, permitiendo gestionar la demanda de energía de la planta de forma automática.

El modelo de negocio de los agregadores se puede dividir - en función de los servicios ofrecidos - entre agregadores con un rol independiente y agregadores con un rol combinado, siendo posible que un mismo agregador oferte ambos servicios. Agregadores independientes son aquellos que actúan con independencia del proveedor usual y del proveedor responsable del servicio de balance. Los agregadores con un rol combinado actúan también como suministradores, lo que reduce la complejidad del servicio. Además de las ventajas por participar en los mecanismos de DSM, la agregación conlleva una ventaja económica, ya que la instalación de estos dispositivos genera un ahorro en el costo de la potencia contratada. Esta actividad es, a la vez, complementada con el uso de sistemas de almacenamiento para reducir la energía proveniente de la red cuando el mercado presenta mayores precios o con un modelo de economía de colaboración como son los prosumidores (consumidores que hacen parte activa de la creación de productos de consumo y servicios de amplio impacto).

Por último, el mercado creado por la ordenanza de cargas interrumpibles fue puesto en marcha en 2012 con el objetivo de reducir el impacto de la energía eólica y aumentar la seguridad del sistema. $\mathrm{AbLaV}$ considera cargas interrumpibles a aquellas unidades de consumo que pueden reducir la demanda de forma programada y segura bajo petición del operador de la red de transmisión. Para ello, regula las condiciones bajo las cuales las industrias intensivas en el uso de energía pueden reducir su nivel de demanda durante periodos cortos de tiempo (cuartos de hora) a cambio de una compensación económica. En 2016, la ordenanza fue modificada para reducir sus requisitos y flexibilidad, aumentando la participación en el esquema, ya que hasta 2016 solo se concretaron seis contratos con cuatro empresas [29]; entre estos cambios destacan: la reducción de la carga mínima disponible de $50 \mathrm{MW}$ a $5 \mathrm{MW}$, la reducción de precio máximo de la capacidad de 2500 Eur/MW y mes a 500 Eur/MW y semana, la convocatoria de licitaciones semanal (antes mensual), los requisitos mínimos de conexión a la red de 110 kV a 20 kV, entre otros [30].

\subsection{Gestión de la demanda en Chile}

A partir de la década del 2000, el sector energético chileno experimenta sustantivas modificaciones legislativas que buscan adaptar y modernizar el marco regulatorio para hacer frente a las nuevas necesidades del país, esto es, a una permanente evolución tecnológica y a un nuevo contexto energético nacional, regional y mundial. Entre 2004 y 2005 entran en vigencia la Ley Corta I (19.940) y la Ley Corta II (20.018), las cuales incorporan por primera vez la definición y promoción de los Medios de Generación de Energías Renovables no Convencionales. Luego, en el 2008, es promulgada la Ley 20.257 que obliga a abastecer un $10 \%$ de la demanda mediante inyecciones provenientes de ERNC, sufriendo en el 2013 una modificación con objeto de alcanzar un $20 \%$ de la demanda abastecida por ERNC en el 2025 (Ley 20.698). Como resultado de estas reformas y 
del amplio potencial ERNC, en marzo de 2018 la capacidad instalada de los sistemas eléctricos chilenos (sistema eléctrico nacional, sistema eléctrico de Aysén y sistema eléctrico de Magallanes) alcanzan 23 534.7 MW, de los cuales 4 558.65 MW corresponden a ERNC. De ellos, 2 447.42 MW corresponden a sistemas solares fotovoltaicos y $1610.55 \mathrm{MW}$ a sistemas eólicos [33]. Asimismo, continúan sumándose nuevos proyectos con base en fuentes renovables de energía variables, se trata de alrededor de 1125 MW sobre energía eólica y solar PV que actualmente se encuentran "en pruebas" y "en construcción", y a los cuales podrían sumarse otros 25017 MW provenientes de proyectos que ya cuentan con calificación ambiental (RCA) favorable [33].

Como en el caso alemán, uno de los principales impactos de la incorporación de las ERNC es el requerimiento de flexibilidad o capacidad del sistema eléctrico para responder a los cambios en el equilibrio de oferta y demanda de manera oportuna, así como el desarrollo de nueva infraestructura para transportar esta energía desde los puntos de generación hasta los de consumo. Además de las ERNC, Chile es país líder en la industria minera, donde estudios de la aplicación de DSM concluyen que es una excelente herramienta económica para controlar la demanda de electricidad debido al alto impacto que podría tener en los costos operacionales [34]. Esto se encuentra condicionado a una correcta caracterización de la demanda como una medida clave para implementar planes de uso eficiente de la energía y capacidad, además de que es necesario crear un mercado secundario con reglas claras y que ofrezca incentivos para aquellos consumidores que empleen DSM [35].

Siendo conscientes de estas dificultades, se han comenzado a mostrar los primeros avances desde el punto de vista de la política energética. La hoja de ruta del Ministerio de Energía chileno propuesta hacia 2050 promueve la producción descentralizada y gestión activa de la demanda, puesto que los mecanismos de gestión de la demanda en conjunto con las aplicaciones de redes inteligentes tienen como finalidad entregar flexibilidad y elasticidad [36], pero también pueden incrementar la eficiencia, competitividad y sostenibilidad del sistema en su conjunto. En esta misma línea, Energía 2050 ha fijado como meta que al 2050 el sector público, comercial y residencial aprovechen su potencial de generación distribuida y gestión de la demanda eléctrica [36]; en consecuencia, se espera que en los próximos años el sistema energético chileno experimente una creciente implementación de DSM.

Para integrar los servicios de DSM dentro del mercado chileno, en 2016 es promulgada la Ley que "establece un Nuevo Sistema de Transmisión Eléctrica y crea un Organismo Coordinador Independiente del Sistema Eléctrico Nacional” (Ley 20.936); con esta legislación, se crea el nuevo marco regulatorio para los consumidores que prestan sus recursos técnicos, ello con objeto de preservar la seguridad del servicio energético en el sistema a partir de los SSCC. Conviene destacar que esta ley también incorpora por primera vez la definición de los sistemas de almacenamiento de energía y equipamiento tecnológico, siendo capaz de retirar energía desde el sistema eléctrico, transformarla en otro tipo de energía (química, potencial, térmica, entre otras) y almacenarla con el objetivo de inyectarla nuevamente al sistema eléctrico. Así, se contribuye a la seguridad, suficiencia o eficiencia económica del sistema, viabilizando que los sistemas de almacenamiento presten flexibilidad para el sistema y sean instrumento de DSM.

En noviembre de 2018, el diario oficial publicó la Ley 21.118 que modifica la Ley General de Servicios Eléctricos, ello con el fin de incentivar el desarrollo de las generadoras residenciales. 
Esta modifica los artículos 149 bis y 149 ter de la Ley 20.571, estableciendo que los usuarios finales sujetos a fijación de precios que dispongan su propio consumo de equipamiento de generación por medios renovables no convencionales o de instalaciones de cogeneración eficiente, de manera individual o colectiva, tendrán derecho a inyectar la energía que generan a la red de distribución. Pueden operar de esta forma conjuntos habitacionales como, por ejemplo, condominios, con un límite de generación donde la capacidad instalada por cada inmueble o instalación de un cliente o usuario final no podrá superar los 300 kilowatts. Las concesionarias de servicio público dispondrán de un contrato con los participantes, definiendo el equipamiento de generación del usuario final y sus características técnicas, capacidad instalada de generación y la opción tarifaria, así como destino de remanentes no descontados. De igual forma, se definen los remanentes de inyecciones de energía que, transcurrido el plazo señalado en el contrato, no hayan sido descontados; así, podrán a voluntad de clientes ser descontados de los cargos por suministro eléctrico correspondientes a inmuebles o instalaciones de propiedad del mismo cliente [37].

Otra de las modificaciones legales y que debería ingresar al Congreso en el presente 2019 es la Ley Miscelánea de Perfeccionamiento del Sector, la cual se dividirá en dos proyectos: el Proyecto de Ley Perfeccionamiento Transmisión y el Proyecto de Ley de Flexibilidad. El primer proyecto busca incorporar herramientas regulatorias que aporten flexibilidad a la ejecución y planificación de la transmisión, incorporando desarrollos tecnológicos de manera eficiente y que permitan aprovechar de una mejor manera los recursos del país. Busca también acortar los plazos y procedimientos para que la generación y la demanda puedan acceder y utilizar las redes eléctricas. El segundo proyecto de flexibilidad tiene como principal objetivo generar las condiciones para que el desarrollo del sistema eléctrico en el mediano y largo plazo sea seguro, eficiente y sostenible, en particular para la creciente inserción de las energías renovables. Se plantea también el desafío jurídico y técnico, donde se debe trabajar por encontrar un justo equilibrio entre la entrega de certeza de señales de largo plazo a los agentes, con marcos normativos que moldeen los espacios para reaccionar a tiempo frente a los cambios. Se tiene en cuenta la incorporación, en el caso de la potencia inicial, de sistemas de almacenamiento, además de incluir consideraciones por indisponibilidad forzada, la relación entre el almacenamiento y la demanda de punta y la determinación de potencia inicial renovable en función del tipo de suministro energético. Dentro de los principales desafíos se encuentra la metodología en la asignación de potencia, destacándose la necesidad de trabajar en elaborar políticas públicas para perfeccionar y modernizar el mercado eléctrico [38].

En la Figura 3, se muestra un paralelismo entre la experiencia alemana y la chilena en términos del desarrollo y aplicación de DSM y DR. Se caracterizan ambos casos por una columna vertebral que retrata una historia similar originada por el aumento en la penetración de energías renovables; el caso alemán presenta mayor anticipo y, por lo tanto, ofrece al caso chileno la posibilidad de acoger iniciativas que han logrado fomentar el uso de esta herramienta.

\section{Discusión}

Los SSCC desempeñan un rol fundamental para enfrentar la incorporación de ERNC variables en la matriz energética chilena, puesto que permiten la utilización de recursos disponibles en el sistema con objeto de preservar la seguridad del servicio eléctrico y, al mismo tiempo, procurando garantizar la operación más económica para el conjunto de las instalaciones. 


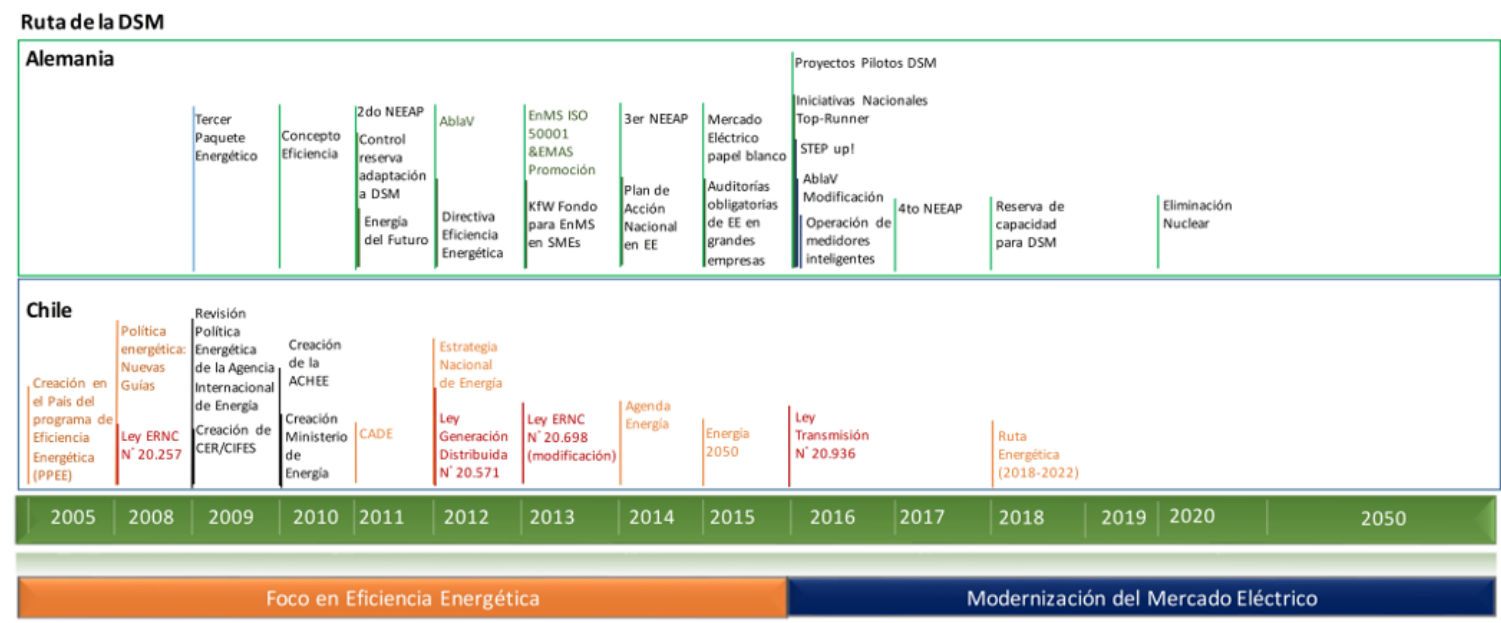

Figura 3. Evolución energética para implementar la DSM en Alemania y Chile.

Fuente: elaboración propia basada en [9].

Si bien se han identificado importantes avances en términos de la discusión e intención de incorporar al sector demanda en un mercado de SSCC, estas iniciativas aún no han visto la luz en su totalidad en Chile. La provisión de reservas de regulación de frecuencia de apertura a DER (Recursos Energéticos Distribuidos) no está exenta de costo para los TSO (Operadores de Sistemas de Transmisión). Los costos de aprendizaje como TSO tendrán que implementar nuevos procedimientos para intercambiar información con diferentes jugadores, y los costos de transacción están presentes. Estos costos deben evaluarse para equilibrarlos con los beneficios potenciales. Sin embargo, dada la creciente participación de las energías renovables intermitentes, los TSO, los reguladores y los gobiernos deben anticipar estos problemas de flexibilidad y explorar opciones para abrir mercados a los nuevos participantes, ello a fin de garantizar una fase de prueba adecuada y gestionar la transición sin problemas antes de verse obligados a hacerlo. Abrir el mercado a nuevos actores, como los agregadores, no debería significar introducir una gran complejidad en los diseños del mercado, lo que podría generar incertidumbre y obstaculizar las inversiones. Se pueden crear excepciones a las reglas o esquemas complementarios dirigidos específicamente a los DER para fomentar las inversiones, pero, en última instancia, se debe implementar un diseño de mercado unificado para obtener reservas al menor costo posible.

Aunque agregadores independientes podrían incrementar la competencia en el mercado y abrir la puerta a nuevos competidores, la falta de un marco claro que regule tanto el rol de los agregadores como las relaciones entre agregadores responsables del servicio de balance y los proveedores de energía hace muy difícil este modelo de negocio. Lo anterior se debe a la necesidad de contar con reglas claras para el servicio de balance, las compensaciones financieras para los proveedores y en la transferencia de datos. La experiencia alemana muestra que modelos de negocio basados en agregadores con un rol combinado podrían ser más compatibles con el diseño de mercado chileno [39].

Transactive Energy (TE), per-to-per (P2P) y control-based approach (CBA) son tres alternativas para abordar los problemas asociados a los AS [40]. TE propone una solución basada en un mercado para la administración de pequeños recursos energéticos distribuidos, servicios de almacenamiento y otras reservas de frecuencia en el nivel de distribución. P2P es un modelo emergente 
basado en la economía colaborativa y permite a los agentes comercializar directamente sus recursos sin la intervención del operador del sistema. Finalmente, CBA considera la agrupación de prosumidores mediante la figura de los subagregadores, los cuales ofertan los recursos ante un agregador. Además, propone un nuevo mecanismo denominado "Ancillary Services 4.0: A Top-to-Bottom Control-Based Approach for Solving Ancillary Services Problems in Smart Grid”, el cual se basa en dos principios básicos: primero, cada prosumidor es controlado por un pequeño administrador local; mientras que el segundo es el tratamiento de la operación de los problemas del sistema de manera desagregada en términos espacio temporales. Todas estas alternativas buscan obtener el máximo provecho - en términos de SSCC — de los "prosumers" de energía localizados en el sector de distribución, sean estos residenciales o industriales; además, integrando la penetración de la electromovilidad.

Con objeto de superar los desafíos que impone el sector eléctrico chileno, cabe destacar la necesidad de un marco regulatorio que abra las puertas para la participación de todos los consumidores industriales que puedan implementar DSM, ello a través de reglas claras e incentivos reales, ya sea de manera directa o a través de terceros. Si se observa el caso alemán, no basta con tener mercados con la opción para que las industrias ofrezcan su potencial de DSM, sino que es necesario un espacio para industrias cuyo negocio principal no es la generación de energía. El spot-market y el mercado de reservas fueron creados con empresas generadoras de energía en mente, y si no se desarrolla un proceso de acompañamiento a la industria productiva no es de esperarse que esta decida participar en estos. Reglas más laxas para la industria productiva permitirían que se integre a ofrecer su potencial de respuesta de demanda durante el proceso de transición a mayores penetraciones de ERNC, y no apenas en algunas décadas después cuando las plantas de generación de energía convencionales sean dadas de baja.

La creación de un mercado propio para competencia entre iguales, como el creado por la $\mathrm{AbLaV}$ en Alemania, sería un paso importante para llevar los potenciales de DSM de la industria chilena al sistema eléctrico; sin embargo, en caso de querer concebir algo así, es importante que se piense en reglas que permitan participar a la industria productiva con altos potenciales de DSM y no solo a grandes industrias. Para ello, será necesario que se estudien aspectos técnicos que pueden variar considerablemente en función del equipamiento y los procesos que se llevan a cabo en plantas como: el nivel de reducción de la carga mínima, la periodicidad de las convocatorias de licitaciones o los requisitos mínimos de conexión a la red, entre otros. Por otro lado, y con el objetivo de adaptar el marco legislativo a la realidad de la economía chilena, se necesitan periodos de revisión más cortos de la normativa (en el caso alemán se necesitaron cuatro años para corregir una normativa que no estaba surtiendo fruto). Si estas condiciones no se dan, valdría la pena concentrarse en un mercado de reservas que sea más vinculante con la industria productiva.

Vale destacar la importancia de las medidas que pueden contribuir a que la industria productiva haga DSM como la implementación de estándares de manejo de energía a la manera del ISO 50001. Como se ha señalado, uno de los requisitos básicos para hacer uso de DSM es la instalación de medidores y el levantamiento de datos. En Alemania, este levantamiento de datos se lleva a cabo mediante certificaciones ISO 50001, puesto que el Gobierno introdujo una serie de políticas para incrementar el número de certificaciones como extensiones de hasta el $99 \%$ de los impuestos de la energía [22], [41]. 


\section{Conclusiones}

Este estudio hace una revisión del desarrollo histórico y conceptos básicos de DSM, presentando los avances regulatorios y organizativos de los mercados en términos de DSM en Alemania y Chile. El análisis comparativo muestra los paralelismos entre los casos chileno y alemán, destacando el reto de integrar una creciente cantidad de ERNC, autoconsumo y la carga de vehículos. La capacidad de ambos sistemas para adaptarse a los nuevos retos pasa por el desarrollo de sistemas de gestión de la demanda que reduzcan la rigidez del sistema, aumentando los niveles de eficiencia y competitividad. Para ello, una mirada a los éxitos y desaciertos de la experiencia alemana muestra que conocer los potenciales de DSM es esencial, ya que sin ella no es posible entender la naturaleza de estos retos.

Tras el análisis de la integración de DSM en la industria, quedan patentes las dificultades existentes a la hora de encajar el amplio potencial de DSM tanto en el mercado chileno como alemán; lo anterior se debe a la falta de una verdadera regulación que permita, no solo participar a aquellas industrias con potencial de DSM en los mercados, sino a la capacidad de establecer reglas claras y mecanismos que integren las características de estas. Asimismo, el análisis del caso alemán muestra la necesidad de reducir la complejidad del marco normativo con el objetivo de facilitar la entrada de nuevos actores que dinamizan el mercado, ya sea directamente o a través de terceros.

En relación con la implementación de DSM, un elemento que resulta trascendental e ineludible es la incorporación de infraestructura asociada a las tecnologías de la información y de la comunicación en el nivel de usuarios o clientes finales, así como en toda la red eléctrica. Se aplica lo anterior también en el caso del sector residencial, que en ambos países necesita un estudio para determinar el potencial real disponible y así hacer factible el buen desarrollo de DSM. La disponibilidad de información instantánea, además de registros históricos y series de datos, viabilizan la posibilidad no tan solo de gestionar la demanda según los requerimientos del sistema, sino que abre la puerta a la creación de nuevos mercados asociados. No obstante, la experiencia alemana muestra que desde el punto de vista económico resulta mucho más viable la implementación de ICT en el sector industrial, dado los volúmenes de energía que se manejan.

Finalmente, es importante que la academia contribuya con números concretos de potenciales de DSM de la industria en el caso chileno y que se estudien cuáles son los incentivos necesarios para que la industria productiva nacional se vincule al mercado de SSCC o alguno similar al creado por la $\mathrm{AbLaV}$, el cual se pudiera crear en Chile. Adicionalmente, es importante que se dé acompañamiento a la industria para que vea las ventajas de ofrecer su potencial de DSM o para que se decida a ofrecer este a través de agregadores

\section{Referencias}

[1] W. S. Tan, M. Y. Hassan, M. S. Majid, y H. Abdul, “Optimal distributed renewable generation planning: A review of different approaches", Renewable and Sustainable Energy Reviews, vol. 18, pp. 626-645, 2013. https:// doi.org/10.1016/j.rser.2012.10.039个236

[2] R. Passey, T. Spooner, I. MacGill, M. Watt y K. Syngellakis, "The potential impacts of grid- connected distributed generation and how to address them: A review of technical and non-technical factors", Energy Policy, vol. 39, n. o 
10, pp.6280-6290, 2011.https://doi.org/10.1016/j.enpol.2011.07.027个236

[3] M. Paulus y F. Borggrefe, "The potential of demand-side management in energy-intensive industries for electricity markets in Germany", Applied Energy, vol. 88, n. o 2, pp. 432-441, 2011. https : / doi.org/10.1016/j. apenergy. $2010.03 .017 \uparrow 236,242$

[4] P. Warren, "The use of systematic reviews to analyse demand-side management policy", Energy Efficiency, vol. 7, n. o 3, pp. 417-427, 2014. https: / / doi.org/10.1007/s12053-013-9230-x $\uparrow 236$

[5] C. Kuzemko, C. Mitchell, M. Lockwood, y R. Hoggett, "Policies, politics and demand side innovations: The untold story of Germany's energy transition", Energy Research and Social Science, vol. 28, pp. 58-67, 2017. https: //doi.org/10.1016/j.erss.2017.03.013个237, 240

[6] C. W. Gellings, "The concept of demand-side management for electric utilities", Proceedings of the IEEE, vol. 73, n. o 10, pp. 1468-1470, 1985.https://doi.org/10.1109/PROC.1985.13318个237

[7] P. Warren, "A review of demand-side management policy in the UK", Renewable and Sustainable Energy Reviews, vol. 29, pp. 941-951, 2014. https://doi.org/10.1016/j.rser.2013.09.009个237, 238

[8] Z. Ming, X. Song, M. Mingjuan, L. Lingyun, C. Min, y W. Yuejin, "Historical review of demand side management in China: Management content, operation mode, results assessment and relative incentives", Renewable and Sustainable Energy Reviews, vol. 25, pp.470-482, 2013. https://doi.org/10.1016/j.rser.2013.05.020 $\uparrow 237$

[9] J. Valdes, A. Poque, L. Ramírez, Y. Masip y W. Dorner, "Industry, flexibility, and demand response: Applying German energy transition lessons in Chile", Energy Research and Social Science, 2019. https: //doi.org/ $10.1016 /$ j.erss.2019.03.003个238, 239, 246

[10] A. F. Meyabadi y M. H. Deihimi, "A review of demand-side management: Reconsidering theoretical framework", Renewable and Sustainable Energy Reviews, vol. 80, pp. 367-379, 2017. https://doi.org/10.1016/j. rser.2017.05.207个238

[11] R. Sharifi, S. H. Fathi, y V. Vahidinasab, "A review on Demand-side tools in electricity market", Renewable and Sustainable Energy Reviews, vol. 72, pp. 565-572, 2017. https://doi.org/10.1016/j.rser.2017. $01.020 \uparrow 238$

[12] M. H. Albadi y E. F. El-Saadany, "A summary of demand response in electricity markets", Electric Power Systems Research, vol. 78, n. o 11, pp. 1989-1996, 2008. https://doi.org/10.1016/j.epsr.2008.04.002个 238

[13] A. Pina, C. Silva y P. Ferrão, "The impact of demand side management strategies in the penetration of renewable electricity", Energy, vol. 41, n. o 1, pp. 128-137, 2012. https://doi.org/10.1016/j.energy.2011. $06.013 \uparrow 238$

[14] C. Bergaentzlé, C. Clastres y H. Khalfallah, "Demandside management and European environmental and energy goals: An optimal complementary approach", Energy Policy, vol. 67, pp. 858-869, 2014. https ://doi.org/ $10.1016 / j$.enpol.2013.12.008 238

[15] International Energy Agency (IEA), "Energy Policies of IEA Countries. Germany 2007 Review”, 2007. $\uparrow 239$

[16] International Energy Agency (IEA), "Germany. Energy System Overview", 2017. $\uparrow 239$

[17] Bundesministerium für Wirtschaft und Energie, "Renewable Energy Sources Act (EEG 2017)", 2017. $\uparrow 239$

[18] O. Borne, K. Korte, Y. Perez, M. Petit y A. Purkus, "Barriers to entry in frequencyregulation services markets: Review of the status quo and options for improvements", Renewable and Sustainable Energy Reviews, vol. 81, pp. 605-614, 2018. https://doi.org/10.1016/j.rser.2017.08.052个240

[19] T. Muller y D. Most, "Demand Response Potential: Available when Needed?", Energy Policy, vol. 115, pp. 181-198, 2018. https://doi.org/10.1016/j.enpol.2017.12.025个240

[20] P. Schott, J- Sedlmeir, N. Strobel, T. Weber, G. Fridgen y E. Abele, "A generic data model for describing flexibility in power markets", Energies, vol. 12, 2019. https://doi.org/10.3390/en12101893个240

[21] A. L. Klingler, "Self-consumption with PV+Battery systems: A market diffusion model considering individual consumer behaviour and preferences", Applied Energy, vol. 205, pp. 1560-1570, 2017. https://doi.org/ $10.1016 / j$.apenergy.2017.08.159个240

[22] Bundesministerium für Wirtschaft und Energie, "Making more out of energy. National Action Plan on Energy Efficiency", Federal Ministry for Economic Affairs and Energy, 2014. $\uparrow 240,247$

[23] P. Bertoldi, P. Zancanella, B. Boza-Kiss, Demand response status in EU Member States. Luxembourg: Publications Office, 2016. $\uparrow 241$

[24] K. Wohlfarth, M. Klobasa y A. Esser, "Setting course for demand response in the service sector", Energy Efficiency, vol. 12, n. o 1, pp. 327-341, 2019. https://doi.org/10.1007/s12053-018-9728-3个241 
[25] R. Seidl, T. von Wirth y P. Kruetl, "Social acceptance of distributed energy systems in Swiss, German, and Austrian energy transitions", Energy Research and Social Science, vol. 54, pp. 117-128, 2019. https://doi . org/10.1016/j.erss.2019.04.006个241

[26] N. Krzikalla, S. Achner y S. Brühl, "Möglichkeiten zum Ausgleich fluktuierender Einspeisungen aus Erneuerbaren Energien”, Büro für Energiewirtschaft und technische Planung GmbH im Auftrag des Bundesverbandes Erneuerbare Energien, Aachen, Germany, Studie im Auftrag des Bundesverbandes Erneuerbare Energie, 2013. $\uparrow 241$

[27] International Organization for Standardization, "ISO Survey 2016", 2017. [En línea]. Disponible en: https://www.iso.org/the-iso-survey.html. $\uparrow 242$

[28] J. Kiljander et al., "Residential Flexibility Management: A Case Study in Distribution Networks", IEEE ACCESS, vol. 7, 2019. https://doi.org/10.1109/ACCESS.2019.2923069个242

[29] Deutsche Energieagentur, "Roadmap Demand Side Management: Industrielles Lastmanagement für ein zukunftsfähiges Energiesystem", Deutsche Energieagentur, Berlin, 2016. $\uparrow 242,243$

[30] Deutsche Bundesregierung, "Verordnung über Vereinbarungen zu abschaltbaren Lasten (Verordnung zu abschaltbaren Lasten - AbLaV)", 2016. $\uparrow 242,243$

[31] B. Bayer, "Current Practice and Thinking with Integrating Demand Response for Power System Flexibility in the Electricity Markets in the USA and Germany", Curr Sustainable Renewable Energy Rep, vol. 2, n. o 2, pp. 55-62, 2015. https://doi.org/10.1007/s40518-015-0028-7个242

[32] 50Hertz Transmission GmbH, Amprion GmbH, TenneT TSO GmbH, TransnetBW GmbH, "Regelleistung.net: Internetplattform zur Vergabe von Regellesitung.", Regelleistung.net, 2018. [En línea]. Disponible en: https://www.regelleistung.net/ext/ $\uparrow 242$

[33] Comisión Nacional de Energía de Chile, "Reporte Mensual ERNC CNE marzo 2018”, Comisión Nacional de Energía, Vol. 19, 2018. $\uparrow 244$

[34] S. Díaz, E. Gil, A. Palacios, "Demand side management, an imperative asset for the mining industry", En ENERMIN 2016: 3rd International Seminar on energy Management in Mining, Santiago de Chile, 2016, p. 10. $\uparrow 244$

[35] V. J. Martinez y H. Rudnick, "Design of demand response programs in emerging countries", Power System Technology (POWERCON), En IEEE International Conference on, 2012. https:// doi.org/10.1109/ PowerCon.2012.6401387 244

[36] Ministerio de Energía de Chile, "Energía 2050: Política Energética de Chile”, 2015. $\uparrow 244$

[37] Comisión de Minería y Energía Cámara de Diputados "Agenda Legislativa 2019. Ruta Energética 2018-2022. Liderando la modernización con sello ciudadano", 2019. $\uparrow 245$

[38] Ministerio de Energía, "Ley 21.118”. Diario Oficial de la República de Chile, 2018. $\uparrow 245$

[39] R. Verhaegen y C. Dierckxsens, "Existing business models for renewable energy aggregators". [En línea]. Disponible en: http://bestres.eu/wp- content/uploads/2016/08/BestRES_Existing-business-models-for-REaggregators.pdf $\uparrow 246$

[40] G. De Zotti, S. A. Pourmousavi, H. Madsen y N. Kjolstad, "Ancillary Services 4.0: A Top-to-Bottom ControlBased Approach for Solving Ancillary Services Problems in Smart Grids", IEEE Access, vol. 6, 2018. https: //doi.org/10.1109/ACCESS.2018.2805330个246

[41] G. F. Siciliano, "Energy management policies and programs from around the globe". [En línea]. Disponible en: https://www.unido.org/sites/default/files/201404/7.Energy_policies_related_to_energy_management__Sicilliano__0.pdf $\uparrow 247$

\section{Axel Poque}

Ingeniero civil en electricidad. Ayudante de Investigación en el Proyecto BMBF150075: "Increasing renewable energy penetration in industrial production and grid integration through optimized chp energy dispatch scheduling and demand side management", financiado por el Ministerio de Educación e Investigación de Alemania (BMBF) y la Comisión Nacional de Investigación Científica y Tecnológica de Chile (CONICYT).

Correo electrónico: axel.poque.g@ mail.pucv.cl 


\section{Meyli Valín}

Dra. Ingeniera mecánica e investigadora en el Proyecto BMBF150075: "Increasing renewable energy penetration in industrial production and grid integration through optimized chp energy dispatch scheduling and demand side management", financiado por el Ministerio de Educación e Investigación de Alemania (BMBF) y la Comisión Nacional de Investigación Científica y Tecnológica de Chile (CONICYT).

Correo electrónico: meyli.valin@ pucv.cl

\section{Luis Ramirez}

Investigador de la Technische Hochschule Deggendorf en Alemania con doctorado en recursos naturales y maestría de ingeniería en medio ambiente y manejo de recursos naturales de la Universidad de Ciencias Naturales de Viena (BOKU). Experto en la modelación espacio-temporal de sistemas de energías renovables y miembro del equipo del proyecto BMBF150075: "Increasing renewable energy penetration in industrial production and grid integration through optimized chp energy dispatch scheduling and demand side management", financiado por el Ministerio de Educación e Investigación de Alemania (BMBF) y la Comisión Nacional de Investigación Científica y Tecnológica de Chile (CONICYT).

Correo electrónico: luis.ramirez-camargo@th-deg.de

\section{Javier Valdes}

Doctor en Ciencias Económicas y Empresariales por las Universidades de Ferrara y Parma, actualmente trabaja en la universidad Technische Hochschule Deggendorf como investigador del proyecto BMBF150075: "Increasing renewable energy penetration in industrial production and grid integration through optimized chp energy dispatch scheduling and demand side management", financiado por el Minis-terio de Educación e Investigación de Alemania (BMBF) y la Comisión Nacional de Investigación Científica y Tecnológica de Chile (CONICYT). Anteriormente ha sido investigador visitante en el Instituto Leibniz de Estudios de Europa del Este y Sudeste (IOS) y en la Universidad Nacional de Educación a Distancia (UNED), e investigador asociado del Grupo de Economía Política Internacional y de la Energía en la UNED.

Correo electrónico: javier.valdes@th-deg.de

\section{Yunesky Masip}

Dr. Ingeniero mecánico y profesor Auxiliar de la Escuela de Ingeniería Mecánica de la Pontificia Universidad Católica de Valparaíso (PUCV). Investigador Responsable del Proyecto BMBF150075: "Increasing renewable energy penetration in industrial production and grid integration through optimized chp energy dispatch scheduling and demand side management", financiado por el Ministerio de Educación e Investigación de Alemania (BMBF) y la Comisión Nacional de Investigación Científica y Tecnológica de Chile (CONICYT). Además, Director de Vinculación con el Medio e Internacionalización del Proyecto Ingeniería 2030 de la Facultad de Ingeniería PUCV y financiado por CORFO-Chile. Correo electrónico: yunesky.masip@pucv.cl 